The Image of Madness 


\section{The Image of Madness}

The Public Facing Mental IIIness and Psychiatric Treatment

Editors José Guimón, Geneva

Werner Fischer, Geneva

Norman Sartorius, Geneva

36 figures and 34 tables, 1999

KARGER $\begin{aligned} & \text { Basel·Freiburg } \cdot \text { Paris } \cdot \text { London } \cdot \text { New York } \\ & \text { New Delhi } \cdot \text { Bangkok } \cdot \text { Singapore } \cdot \text { Tokyo } \cdot \text { Sydney }\end{aligned}$ 


\section{- n \\ José Guimón \\ Werner Fischer \\ Norman Sartorius}

Department of Psychiatry (HUG)

2, chemin du Petit-Bel-Air

CH-1225 Chêne-Bourg/Geneva (Switzerland)

Library of Congress Cataloging-in-Publication Data

The image of madness / editors, José Guimón, Werner Fischer, Norman Sartorius.

Includes bibliographical references and index.

1. Mental illness - Public opinion. 2. Mental illness - Chemotherapy - Public opinion. I. Guimón, J.

II. Fischer, Werner. III. Sartorius, N.

[DNLM: 1. Mental Disorders - psychology. 2. Attitude to Health. 3. Public Opinion. WM 31 I31 1999] RC455.2.P85I46 $1999362.2-$ de21

ISBN 3-8055-6846-0 (hardcover: alk. paper)

Drug Dosage. The authors and the publisher have exerted every effort to ensure that drug selection and dosage set forth in this text are in accord with current recommendations and practice at the time of publication. However, in view of ongoing research, changes in government regulations, and the constant flow of information relating to drug therapy and drug reactions, the reader is urged to check the package insert for each drug for any change in indications and dosage and for added warnings and precautions. This is particularly important when the recommended agent is a new and/or infrequently employed drug.

All rights reserved. No part of this publication may be translated into other languages, reproduced or utilized in any form or by any means electronic or mechanical, including photocopying, recording, microcopying, or by any information storage and retrieval system, without permission in writing from the publisher.

(C) Copyright 1999 by S. Karger AG, P.O. Box, CH-4009 Basel (Switzerland)

www.karger.com

Printed in Switzerland on acid-free paper by Reinhardt Druck, Basel

ISBN 3-8055-6846-0 


\section{Contents}

VIII Introduction

Social Representations of Mental Illness

1 Attitudes toward Mental Illness among the General Public and Professionals, Social Representations and Change Eker, D.; Öner, B. (Ankara)

13 Mental Illness as Metaphor Finzen, A.; Hoffmann-Richter, U. (Basel)

20 Social Representations of Mental Illness among the Public Angermeyer, M.C.; Matschinger, H. (Leipzig)

29 The Image of Mental Illness in Switzerland Brändli, H. (Marsens)

38 Public Attitudes towards Deviant Situations in Daily Life: Intervention Proposed

Goerg, D.; Fischer, W.; Zbinden, E.; Guimón, J. (Geneva)

56 The General Public's Cognitive and Emotional Perception of Mental Illnesses: An Alternative to Attitude-Research

Hillert, A. (Prien am Chiemsee); Sandmann, J.; Ehmig, S.; Weisbecker, H.; Kepplinger, H.M.; Benkert, O. (Mainz)

72 'Cooperating', 'Fighting against' or 'Letting Go' in the Therapeutic Context: Social Logic of Parents with Psychologically Disturbed Children

Schurmans, M.-N. (Geneva); Duruz, N. (Fribourg/Lausanne) 
85 Differentiating between the Professions of Psychologists and Psychiatrists: A Field Study in Vizcaya

Yllá, L.; Hidalgo, M.S. (Bilbao)

Changing Attitudes towards Mental Illness and Destigmatization

96 One of the Last Obstacles to Better Mental Health Care: The Stigma of Mental Illness

Sartorius, N. (Geneva)

105 Public Education for Community Care: A New Approach Wolff, G.; Pathare, S.; Craig, T.; Leff, J. (London)

118 Combatting the Alienation Experienced by People with Mental IIIness Warner, R. (Boulder, Colo.)

129 Group Therapy and Attitudinal Changes to Mental Illness in Medical Students

Yllá, L.; González-Pinto, A. (Lejona)

138 Stigmatization and Destigmatization: The Point of View of Psychiatric Patients and Their Families

Pont, M. (Lausanne)

Attitudes towards Psychiatric Treatment and Compliance

143 Use and Misuse of Pharmacological Substances: The Question of Noncompliance

Guimón, J.; Fischer, W.; Goerg, D.; Zbinden, E. (Geneva)

152 The Public's Attitude towards Drug Treatment of Schizophrenia Angermeyer, M.C.; Matschinger, H. (Leipzig)

162 Determining Factors and the Effects of Attitudes towards Psychotropic Medication

Fischer, W.; Goerg, D.; Zbinden, E.; Guimón, J. (Geneva)

187 Attitudes towards Psychotropic Medication among Medical Students Hillert, A. (Prien am Chiemsee)

197 Measuring Attitudes toward Psychiatric Medication among Persons with Serious Mental Illness

Draine, J. (Philadelphia, Pa.)

208 Psychoeducational Groups in Schizophrenic Patients Eguiluz, I.; González Torres, M.A. (Bilbao); Guimón, J. (Geneva)

216 Neuroleptics: The Point of View of Consumers and Their Families Spagnoli, A. (Lausanne) 
222 The Role of Communication and Physician-Patient Collaboration: Enhancing Adherence with Psychiatric Medication

DiMatteo, M.R. (Riverside, Calif.)

231 Rethinking the Problem of Noncompliance in Chronic Mental Illness Thorne, S.E. (Vancouver, B.C.)

239 Author Index

240 Subject Index 


\section{Introduction}

Negative moral judgments seem to have been a constant fixture in the way societies and cultures have regarded groups displaying deviant behavior. This is particularly true of the mentally ill who, during the early nineteenth century, underwent classification into categories susceptible to psychiatric treatment. 'Medicalizing' this particular form of deviancy has not led to an alleviation, or even a neutralization, of the negative responses it inspires. On the contrary, stigmatization has spread to psychiatry itself which was made responsible for controlling and correcting its manifestations. This remains true today since the term 'psychiatrization', despite some semantic modifications, is unanimously defined as a derogatory description of behavior, attitude and thought.

Psychiatric deinstitutionalization, beginning in the 1950s, did not merely open a door onto the outside world and society; it multiplied the contacts between the mentally ill and persons considered to be normal. Stigmatization which had originally centered on an entity, a concept - madness - and on its almost exclusive domain - the psychiatric hospital, thus shifted, influencing public conduct towards the mentally ill in the community, relations with and attitudes towards them. This passage into open society conditioned other transformations, for example the creation of differentiated negative stereotypes resulting from specific behavior, specific psychopathology. But the most radical stigmatization systematically victimizes schizophrenics who seem to display the greatest number of traits which lead to rejection and social exclusion.

Studies on social representations and public attitudes towards mental illness, on stigmatization and the exclusionary process from which patients suffer, were carried out over this same period in both psychiatry and the social sciences. Two principal orientations emerged to structure perspectives: 'labeling' first theorized by Lemert and Scheff in the 1950s and 1960s and the 
interactionist theory primarily defended by Goffman and Mechanic. All of the studies, the most representative of which are quoted in this volume, report the general prejudice against the mentally ill whatever the context: different cultures, urban or rural regions, care facilities, target audiences or general public.

The present work evaluates the current status of social stereotypes associated with mental illness as well as examining attitudes towards psychiatry in general. It also investigates the changes that have occurred in this area. Our endeavor appears even more timely as the World Psychiatric Association has instigated a program which aims to lessen the stigmatization of the mentally ill and forestall their exclusion from many spheres of life. Finzen reminds us, first of all, of the pivotal role played by schizophrenia in popular stereotypes of mental illness and in the mechanisms of stigmatization. Endowed with occult powers, it is interpreted as a mysterious malediction, bizarre and inexplicable. The schizophrenic suffers from total and absolute vilification as if the whole of his or her being were infected, ravaged by illness.

The idea of the outgrowth of the illness is probably linked to the predominant public image of schizophrenia: a personality disintegrated, split into several disjointed elements, as Angermeyer and Matschinger show in their analysis of the social representations of schizophrenia. Pont also points out that the stigma adds to the burden of the primary disorder to create schizophrenia's secondary illness which violates not only the patient's identity, but distorts the image they have of themselves. The stigma then appropriates all the links to mental illness: discrediting patient associations, care facilities and psychiatric professionals.

The danger represented - or thought to be represented - by psychiatric patients is another fixture of the labeling process. According to various studies, perception of the danger represented by the mentally ill is inversely proportionate to familiarity: the more contact there is, the less mental patients are deemed threatening. Experience with mental illness, either directly or indirectly, also diminishes stereotypes about perceived danger. Brändli draws our attention to the fact that subjects and their entourage tend to confine the social interaction of someone who is mentally ill to the family circle, a restricted network of close friends and the family doctor, in order to avoid embarrassing situations. They appear to validate outright public fears of contact with the mentally ill, reconciling themselves to prejudice and thereby contributing to and reinforcing stereotypes.

It is interesting to note - as Goerg et al. show - that the logic behind reactions to the mentally ill themselves can be discovered in the description of social situations which represent less obviously visible forms of deviant behavior and which are not readily considered to be manifestations of illness. 
Angermeyer and Matschinger reported that the general public does not a priori differentiate between the different psychiatric disorders (schizophrenia, depression and panic disorder) or between the various psychotropic medications prescribed for them. This is, however, not the case when the public is confronted by deviant situations in daily life. Preference is then given to psychiatric intervention, even recourse to psychotropic medication, more often to counter violence than for odd behavior or symptoms of withdrawal. Psychiatry therefore finds its legitimacy in situations that lend themselves most readily to stigmatization.

Yllá and Hidalgo find analogous results in the general public and among psychology students for their representation of the professions of psychiatry and psychology. Psychiatrists were assigned the treatment of the mentally ill and those patients having psychological problems associated with somatic illness. Psychologists were expected to resort exclusively to psychology to resolve difficulties for persons who were in otherwise good physical health. In a more general fashion, Sartorius supports the notion that the social stigmatization of mental patients is reinforced and reproduced in the health-care system. Several indices confirm this: lesser resources allocated to psychiatry than to somatic medicine, more restricted access to treatment, lower standards in psychiatric practice, absence of research which would permit the elaboration of strategies to prevent psychiatric disorders and their effects.

For the study on representations of mental illness, two types of methodology were principally used. In the first instance, the technique of using vignettes elaborated by Star was employed to create a portrait constructed out of the most salient diagnostic features of, for example, schizophrenia, anxiety disorder and affective disorders. The second method of research utilized attitude scales of which the principal and most commonly used is the OMI scale (Opinions about Mental Illness) of Cohen and Struening. This scale, many of whose variations have been formulated and adapted in the context of specific research, present a factorial structure which has shown itself to be stable in different studies.

From a methodological point of view, these two research trends have formed the object of a certain amount of criticism, according to which both the vignettes and the attitude scales confront interview subjects with scenarios based in clinical psychiatry and the institutional management of patients. Answers would therefore be prompted by certain characteristics whose pertinence resides in underlying psychopathology rather than in the logic applied to social interaction and the cultural significance of daily life. Other remarks stress the fact that, among subjects interviewed, few have ever encountered the situations presented in the questionnaire or mentioned in the scales; this could engender hypothetical reactions and attitudes which may diverge very 
strongly from real-life situations. Finally, these methods would give credency to the notion that mental illness is a singular concept that does not include differing mental states.

New research perspectives have been applied and are described in this volume. Hillert et al. consider that the image of mental illness conveyed by clinical vignettes is much too general and lacking in differentiation. They propose clearly distinguishing between cognitive and affective dimensions, thus building profiles which take into account the disparate identities of psychiatric patients. These profiles describe situations which correspond most closely to the distinctions subjects make about different disorders under varying social conditions. Schurmans and Duruz propose abandoning the approach which primarily attempts to assess how the lay public connects to scientific knowledge and mental health professionals. This reorientation should be directed towards examining the compatibility and antagonism that exists between lay knowledge and professional expertise. Therapeutic relationships furnish a prototype. These authors note that 'Therapists should appreciate that all knowledge is elaborated from pre-existing conceptions and knowledge that should be taken into consideration and therefore unveiled.' This is true not only of therapeutic relations, but also of any predicament where disparate theories and knowledge come into confrontation - whether the problem is mental illness itself or compliance, as thoroughly analyzed by Thorne.

Certain articles contained in this volume touch on the question of whether attitudes on, and representations of mental illness and patients have changed or if, on the contrary, we are witnessing the endurance of negative stereotypes. Eker and Öner champion recourse to experimental methods, especially to change attitudes towards the mentally ill. Wolff et al. describe the results of such an experimental study. They note that: 'The results of the follow-up survey suggest that although the public education intervention may have, at best, only a modest effect on knowledge, it is associated with an improvement in overall attitudes and behavior toward the mentally ill. ... However, the education campaign did not in itself lead directly to less fearful attitudes, whereas contact with patients did. It is likely, therefore, that the campaign exerted its effect on overall attitudes indirectly by encouraging contact with patients.' Yllá and González-Pinto observe practically no change in attitudes towards mental patients among medical students. No significant difference was noted between the two types of intervention: theoretical formative and experiential.

According to Eker and Öner, a major reason for the absence of modification in attitudes may reside in the fact that stereotypes and negative attitudes are assimilated at an early stage of socialization. They form part of the very basis of our culture. Thus, effectively, this is not simply a question of isolated 
prejudice. In the study of Fischer et al., it is shown that we are faced with belief systems encompassing not only illness and psychiatric patients, but also psychiatric therapy. Warner views the situation from the same angle. After having analyzed the fundamental aspects of alienation (meaninglessness, powerlessness, normlessness and estrangement from society and work), he shows how these problems may be dealt with to lessen their effects: humanizing and normalizing techniques which empower patients, alternatives to hospitalization, professional activity to promote feelings of worth and self-esteem, consumer-run programs.

Comments made about the general public image of mental illness also apply to opinions and attitudes about psychiatric treatment, particularly psychotropic medication. Angermeyer and Matschinger point to the important hiatus between popular beliefs and current scientific knowledge. Firstly, the public does not differentiate between neuroleptics and other psychotropic drugs (anxiolytics and antidepressants for example) and has very negative attitudes towards them. The notion is widely held that these drugs are addictive or lead to dependency and should only be used as a last resort.

Attitudes towards psychotropics and medication in general seem to be influenced by their own logic, independent of any beliefs about the origin of mental illness or its evolution. The analysis of different therapeutic possibilities reveals, in the study by Fischer et al., a new aspect: bias against and refusal of psychotropics constitute indications of a more general opposition to medicine itself and psychiatry in particular. Effectively, people who refuse psychotropics as a therapeutic option also tend to disregard both individual psychotherapy and family therapy, preferring natural means and relaxation techniques. In this area as well, studies have been carried out on modifications in attitude for health-care professionals such as, for example, that of Hillert et al. among medical students. An altered outlook seems a certainty as students advance through the syllabus. But the question of ascertaining which processes effect these changes should be further investigated.

In improved acceptance of psychotropic medication lies crucial patient compliance; from numerous studies we have learned that it is weak and problematic. The chasm dividing therapeutic conviction from patient behavior seems so wide that the usual recommendations (a relationship of trust, accessible language in communicating with the patient, involvement of the entourage) do not appear to rectify this therapeutic deficit. In contrast, Eguiluz et al. devised a psychoeducational program for groups of schizophrenic patients with the aim of promoting improved compliance in order to decrease risks of relapse. When compared to a control group, those patients in the program were significantly less frequently readmitted to hospital. 
New research directions allow us to better understand this phenomenon. After having analyzed recourse to psychotropics in various countries and the factors significantly associated with their prescription, Guimón et al. place difficulties with compliance in the context of the patient's attitude towards his or her disorder. Thus, poor compliance may be a sign of denial of illness or an insufficient consciousness of it. The patient may stop taking medication to 'test' his or her mental state in the absence of psychopharmacological intervention. Alternatively, the therapist may unconsciously contribute to a patient's lack of compliance. Spagnoli also notes that the benefits of neuroleptics are often better perceived by therapists and a patient's entourage than by patients themselves. She proposes an interpretation of the significance of taking or refusing this medication. Effectively, medication confirms the illness and hints at its severity, constantly reminding a patient - even when he or she is feeling better - of its existence. Additionally, when responsibility for supervision of compliance falls to the entourage, it is symbolic of the denial of a patient's autonomy. Warnings about noncompliance affix themselves to other judgments of inadequacy, thereby corroborating not only psychiatric disorder but also reinforcing social stereotypes.

Draine extends the analysis of the problem of compliance to persons in the patient's entourage, studying their own therapeutic discipline. Therefore, compliance with medication should not be considered merely intrinsic to patients or to a psychiatric disorder. Attitudes towards prescription drugs would thus form part of a collective context which influences - as much as personal prejudice - the decisions patients take with regard to their medication.

Finally, DiMatteo proposes that the patient be implicated as much as possible in the decisions taken by the physician, that the patient be granted the right to be an active partner in issues of consent and informed choice. For these aspects, in analogy to the findings of Schurmans and Duruz on representations parents make of their child's psychiatric disorder, Thorne defines noncompliance not in the terms of a senseless act, but rather as a rational choice based on a patient's knowledge and experience. In this domain, patients also have an obvious expertise. Such questions can no longer be considered as a lack of submission on the patient's part to the physician's greater authority and knowledge; they have become bargaining chips between the therapist and patient in negotiating the different aspects of illness where the management of medication becomes an integral issue.

These changes in perspective concern patient compliance and its relationship to treatment and psychotropic drugs in particular. They are an element of the sea change which would narrow the breach, extensively documented in the literature, between mental illness and normality. Studies and strategies of action to counteract discrimination against the mentally ill have gained 
momentum, not only in medicine and psychiatry, but also in the sociopolitical arena and in the advocacy movement. The goal of these programs is to better educate the general public, increasing community involvement, knowledge and awarness of mental illness throughout the health-care and educational systems as well as changing the laws that sustain, or even reinforce the stigmatization and exclusion which effectively constitute the major obstacle to efforts to develop community medicine and mobilize resources in order to promote an inclusive society and better serve the needs of patients and their families.

\author{
José Guimón \\ Werner Fischer
}

Norman Sartorius 